\title{
DOCTRINA
}

\section{LAS ASOCIACIONES CIVILES COMO ORGANIZACIONES. CONCRECIÓN E INTEGRACIÓN DEL DERECHO CONSTITUCIONAL DE ASOCIACIÓN}

\author{
Virginia S. Bado Cardozo*
}

\begin{abstract}
RESUMEN. En Uruguay, el derecho de asociación se encuentra protegido constitucionalmente y el Estado reconoce a las asociaciones el atributo de la personalidad jurídica en tanto se cumpla con una serie de requisitos formales. Las normas que regulan a las asociaciones son muy específicas $y$ resultan insuficientes para completar las omisiones estatutarias. De forma de integrar las referidas omisiones, en este trabajo se observa a la asociación civil desde la particular visión de la teoría del órgano y se sostiene que ésta no ha de ser una doctrina de aplicación exclusiva del Derecho Público. Por el contrario, se afirma el valor del órgano como formador de la voluntad de la persona jurídica asociación y se examina el ámbito de la competencia como garantía de la separación de poderes en esta institución. Asimismo, comprendiendo que la asociación civil es un fenómeno asociativo afín a la sociedad comercial, es de rigor analizar la posibilidad de integrar el esbozado derecho de asociación con la legislación societaria.
\end{abstract}

PALABRAS CLAVE. Asociación civil. Teoría del órgano. Institución. Competencia. Sociedad comercial.

ABSTRACT. In Uruguay, freedom of association is enshrined in the constitution, and the State recognizes associations as legal entities as long as a number of formal requirements are met. The rules that regulate associations are very specific and fail to make up for omissions in the bylaws. In order to integrate the aforementioned omissions, this paper looks at civil associations from the particular viewpoint of the Organ

* Doctora en Derecho y Ciencias Sociales UDELAR. Doctora en Derecho por la Universidad de Valencia (España). Profesora Adscripto Adjunto de Derecho Comercial UDELAR. Correo electrónico: vbado@derechocomercial.edu.uy. 
Theory of Government and maintains that this doctrine should not be exclusively applied by Public Law. On the contrary, it endorses the value of government bodies as originators of the will of an association as a legal entity and examines the scope of competition as a guarantee of division of powers in this institution. Furthermore, on the understanding that civil societies are an associative phenomenon related to corporations, it is necessary to analyze the possibility of integrating the outlined freedom of association together with corporate legislation.

KEY WORDS. Civil association. Organ Theory of Government. Institution. Competition. Corporations.

\section{REGULACIÓN DEL DERECHO DE ASOCIACIÓN Y DE LA ASOCIACIÓN EN EL DERECHO URUGUAYO}

\section{A. Constitución de la República}

En Uruguay existe un derecho de todas las personas a asociarse, cualquiera sea el objeto que persigan, en tanto no constituyan una asociación ilícita declarada por la ley. Así se encuentra establecido en el artículo 39 de la Constitución vigente. Las asociaciones ilícitas se encuentran definidas en el artículo 1 de la Ley 9936/1940, de 18 de junio ${ }^{1}$.

El derecho de asociación viene siendo reconocido desde la Constitución de 1934, en el artículo $38^{2}$. Luego, en la Constitución de 1952, pasa a ubicarse en el artículo 39 y allí permanece, inalterado, desde entonces, hasta nuestros días ${ }^{3}$.

$1 \quad$ El artículo 1 de la referida Ley establece: «Se consideran asociaciones ilícitas: $1^{\circ}$ Las que difundan ideas contrarias a la forma de gobierno democrático-republicana, adoptada en el primer inciso del artículo 72 de la Constitución.

$2^{\circ}$ Las de carácter político o social, excepción hecha de las de carácter religioso, que en su organización o funcionamiento o directrices o finalidades o provisión de recursos, estén vinculadas a la voluntad de una persona o de un poder extranjero, o de cualquier entidad extraña al pais, en vez de estarlo a la de sus asociados.

$3^{\circ}$ Las constituidas en la República con finalidades de acción política en el exterior, y

$4^{\circ}$ Las que usen enseñas, uniformes, símbolos o saludos que singularicen a partidos, tendencias o entidades políticas extranjeras».

2 El artículo 38 de la Constitución de 1934 dispuso: "Todas las personas tienen el derecho de asociarse, cualquiera sea el objeto que persigan, siempre que no constituyan una asociación ilícita declarada por la Ley".

MARTins entiende que el artículo 173 de la Constitución de $1918^{2}$ contemplaba al derecho de asociación en tanto derecho inherente a la personalidad humana (Martins, Daniel Hugo, Constitución de la República Oriental del Uruguay. Comentada, anotada y concordada, t. 1 [Montevideo: La Ley, 2014], pp. 253 y 254). El artículo 173 de la Constitución de 1918 establecía: "La enumeración de derechos y garantías hecha por la Constitución, no excluye los otros que son inherentes a la personalidad humana o se derivan de la forma republicana de gobierno".

3 Excede las pretensiones de este trabajo reflexionar sobre el derecho constitucional de asociación. No obstante, conviene diferenciarlo del derecho de reunión, derecho constitucionalmente protegido desde la Constitución de 1934 en el artículo articulo 37: "Queda garantido el derecho de reunión pacífica y sin armas. El ejercicio de este derecho no podrá ser desconocido por ninguna autoridad de la República, sino en virtud de una Ley y solamente en cuanto se oponga a la salud, la seguridad y el orden públicos». Este derecho continúa protegido hasta nuestros días (actualmente en el artículo 38 de la Constitución) y su fuente se halla en el artículo 1 de la Ley 2499/1897, de 28 de julio. Pese a ello, el derecho no se incorporó a la Constitución de 1918 y hubo de esperarse hasta la de 1934. 


\section{B. Código Civil}

El derecho de asociación se concreta en el artículo 21 del Código Civil. Esta norma establece que todos los individuos de la especie humana son personas y considera personas jurídicas y por consiguiente capaces de derechos y obligaciones civiles, al Estado, el Fisco, el Municipio, la Iglesia y las corporaciones, establecimientos y asociaciones reconocidas por la autoridad pública.

En lo que refiere a las personas jurídicas, la norma las clasifica en públicas y privadas ${ }^{4}$. El reconocimiento de la personalidad jurídica de las asociaciones civiles viene dado por el Estado, por intermedio del Ministerio de Educación y Cultura, a pedido de la asociación, para cada caso concreto. Se trata, éste, de un reconocimiento administrativo ${ }^{5}$, resultado de un acto administrativo.

\section{Leyes}

Tal vez sea menester comenzar el repertorio de leyes relacionadas con las asociaciones haciendo referencia a la Ley 15.737/1985, de 8 de marzo, norma que ratifica la Convención Americana sobre Derechos Humanos (el denominado "Pacto de San José de Costa Rica»), cuyo artículo 16 reconoce a todas las personas el derecho a asociarse libremente con fines ideológicos, religiosos, políticos, económicos, laborales, sociales, culturales, deportivos o de cualquiera otra índole. El ejercicio de este derecho de asociación sólo puede estar sujeto a las restricciones previstas por la ley que sean necesarias en una sociedad democrática, en interés de la seguridad nacional, de la seguridad o del orden públicos, o para proteger la salud o la moral públicas o los derechos y libertades de los demás. La norma dispone deja a salvo la imposición de restricciones legales, y aun la privación del ejercicio del derecho de asociación, a los miembros de las fuerzas armadas y de la policía.

Existe una ley que se aplica a todas las asociaciones, pero que trata de un asunto tan puntual que de modo alguno puede considerarse una ley reguladora de la asociación. Nos referimos a la Ley 12.284/1956, de 9 de mayo, de Asociaciones Civiles, ley que, pese a la generalidad de su nombre, tiene un único artículo que se limita a autorizar a las asociaciones que cumplan ciertos requisitos a sesionar en segunda convocatoria con el número de asociados que concurra ${ }^{6}$.

La Ley que merece mayor atención es la 15.089/1980, de 9 de diciembre, por la cual se establece que el Ministerio de Educación y Cultura será quien ejercerá la policía administrativa de las asociaciones civiles y de las fundaciones. De acuerdo al artículo 1, los cometidos del Ministerio se reducen a ejercer el control sobre los actos de creación, de funcionamiento, disolución y liquidación. Luego, el artículo 2 le otorga facultades para aplicar sanciones a las asociaciones civiles y fundaciones que incurran en infracciones a las

\footnotetext{
Gambino Crevani, Hugo, Personas jurídicas (Montevideo: Facultad de Derecho, 1958), p. 25.

Berdaguer, Jaime, La sociedad civil jes sujeto de derecho? (Montevideo: FCU, 2007), p. 21.

$6 \quad$ El artículo 1 de esta Ley establece: "Autorízase a las Asociaciones Civiles que a la fecha de la presente ley, gozando de personería jurídica, tengan más de cinco mil asociados, y cuyos Estatutos exijan quórum o mayorías especiales para su reforma - y al solo fin de la aprobación de nuevos procedimientos de modificación de los textos estatutarios - a efectuar, como etapa complementaría del trámite vigente, una segunda convocación del órgano que corresponda, el que podrá sesionar entonces con el número de asociados que concurran, y adoptar resoluciones por mayoría del total de presentes».
} 
normas legales, reglamentarias o estatutarias. La norma establece una serie de sanciones, las que vienen graduadas en orden de importancia, en observación, apercibimiento y multa. La potestad de imponer estas sanciones es propia del Ministerio de Educación y Cultura.

El artículo 3 establece lo que la Ley denomina intervención, medida que es dispuesta por el Poder Ejecutivo, a propuesta del Ministerio de Educación y Cultura, en tanto se acrediten las situaciones de hecho previstas en los numerales 1,2 y $3^{7}$. No se trata de una sanción. De acuerdo al artículo 4, la medida de intervención puede estar a cargo de un veedor, uno o varios coadministradores, o de uno o varios administradores. Todo lo relacionado con sus atribuciones y el plazo de duración de la medida es competencia exclusiva del Poder Ejecutivo, a propuesta del Ministerio de Educación y Cultura.

Existe, luego, la Ley 15.137/1981, de 21 de mayo, de Asociaciones Profesionales ${ }^{8}$ y la Ley 17.777/2004, de 21 de mayo, de Constitución de asociaciones y sociedades agrarias, contratos agrarios colectivos y de integración ${ }^{9}$.

\section{Decretos}

Existen dos decretos con diverso contenido, uno que establece multas para el caso de infracciones a las normas legales, reglamentarias o estatutarias (Decreto 61/2012), que se entiende enmarcado en el Decreto Ley 15.089 referido, y otro que reglamenta principalmente el procedimiento tendente a evitar que las instituciones civiles tengan una denominación igual o similar (Decreto 608/1973).

\section{E. Resoluciones}

Frente a la escasez de leyes y decretos se destaca la abundancia de resoluciones. Emanan de diferentes reparticiones de la Administración Pública (Administración de los Servicios de Salud del Estado, Ministerio de Educación y Cultura, Ministerio de Trabajo y Seguridad Social, Ministerio de Industria, Energía y Minería) o de algunos gobiernos departamentales y refieren a cuestiones específicas que involucran a determinada asociación ${ }^{10}$.

7 El artículo 3, en los numerales referidos, dispone:

«1) Cuando hubiere comprobado actos graves que importaran violación de la ley, de la Reglamentación o del estatuto.

2)Cuando la medida resultare necesaria para proteger el interés público.

3)Cuando la situación de hecho imponga la necesidad de salvaguardar el patrimonio de aquéllas o los bienes morales o materiales que estuvieran a su cargo. En todo caso la intervención no podrá extenderse por más de seis meses, prorrogable por otros seis, por una sola vez. La medida tendrá siempre como finalidad restituir a la institución en el más breve término al cauce normal de su actividad y funcionamiento, o proceder, si ello no fuera posible o aconsejable, a la disolución y liquidación de la misma una vez cancelada su personería jurídica».

8 De acuerdo al artículo 1 de esta norma, las asociaciones profesionales son asociaciones civiles constituidas por trabajadores o empleadores de la actividad privada, que tienen como objeto la promoción, el estudio, la mejora y la defensa de sus respectivos intereses en el ámbito laboral.

9 El primer párrafo del artículo 4 de esta norma, bajo el título «De las asociaciones agrarias», dispone que "Las asociaciones agrarias son aquellas en que la voluntad asociativa se forma por acto constitutivo previsto en documento público o privado y suscrito por los fundadores, con el objeto dispuesto en esta ley".

10 En general tratan de la aprobación de proyectos de convenio entre la Administración y determinada asociación, la autorización de intervenciones sobre las asociaciones, su prórroga y cese, la concesión 
No puede dejar de observarse la Resolución del Poder Ejecutivo, de 21 de septiembre de 1993, por la cual se propone un estatuto denominado «tipo» para las asociaciones civiles.

\section{LAS ASOCIACIONES COMO INSTITUCIONES ORGANIZADAS}

\section{A. El órgano en la teoría de la institución}

Se considera persona jurídica a toda persona constituida por entes públicos, asociaciones privadas o corporaciones de economía mixta que, en virtud de la ley o de un acto administrativo dictado con arreglo a la misma, ha sido investida de la posibilidad de adquirir derechos y de contraer obligaciones ${ }^{11}$. A los efectos de adquirir estos derechos y de contraer esas obligaciones, las personas jurídicas necesitan de órganos. Los órganos de las asociaciones civiles les permiten concentrar los esfuerzos de los asociados y utilizar los bienes comunes para la realización del fin que impone el interés colectivo en juego ${ }^{12}$.

La estructura orgánica de las personas jurídicas, como lo son las asociaciones, es una premisa aceptada por todas las teorías de la personalidad jurídica que la explican como una realidad, ya sean organicistas (SCHAEFFLE y GIERKE), institucionalistas (HAURIOU y RENARD) o basadas en el interés protegido (SALEILLES y FERRARA) ${ }^{13}$. Esta premisa no es compartida por aquellos autores que sostienen que la personalidad jurídica de las asociaciones se explica por la teoría de la ficción.

Entre nosotros, SAYAGUÉs LASO afirma que el concepto de órgano se estudia habitualmente en relación con la administración de las personas públicas, a pesar de que lo correcto sería estudiarla, con carácter general, tanto respecto de personas públicas como privadas ${ }^{14}$. En la misma línea de pensamiento, CAJARville Peluffo sostiene que los conceptos de «persona jurídica», «cargo» y «órgano», como medios instrumentales de su actuación, son admitidos y utilizados en todas las ramas del Derecho, sean públicas o privadas, perteneciendo, en realidad, a la Teoría General del Derecho ${ }^{15}$.

\section{B. Concepto orgánico de asociación}

En los términos expuestos, es comprensible que la mayor parte de las definiciones de asociación refieran al concepto de órgano. Entre nosotros, CoUTURE entiende a la asociación como el "conjunto de personas agrupadas con arreglo a una organización estatutaria, con el objeto de lograr determinados fines materiales o espirituales» ${ }^{16}$. En la doctrina española, GARRIGUes DíAZ-CABAÑete define a la asociación como toda unión voluntaria, duradera y organizada de personas que ponen común sus fuerzas para conseguir un fin determinado ${ }^{17}$.

de autorizaciones de variada índole ante distintas solicitudes efectuadas por ciertas asociaciones y otras cuestiones puntuales, de interés para las asociaciones involucradas.

11 Couture, Eduardo, Juan, Voz «personalidad jurídica», Vocabulario Jurídico (Montevideo: Biblioteca de Publicaciones Oficiales de la Facultad de Derecho y Ciencias Sociales de la Universidad de la República, 1960), p. 466.

12 Gambino Crevani, op. cit., p. 52.

13 Gambino Crevani, op. cit., pp. 52 y ss.

14 Sayagués Laso, Enrique, Tratado de Derecho Administrativo, t.1 (Montevideo: s/e, 1963), p. 192.

15 Cajarville Peluffo, Juan Pablo, Sobre Derecho Administrativo, t.1, $3^{\mathrm{a}}$ ed. (Montevideo: FCU, 2012 ), p. 569.

16 Couture, Voz «Asociación», op. cit., p. 124.

17 Garrigues Díaz-Cabañete, Joaquín, Curso de Derecho Mercantil, t. I, $7^{\text {a }}$ ed. (Madrid: Aguirre, 1976 ), p. 307. 
Broseta Pont agrega que la finalidad de esta unión consiste en alcanzar un fin de carácter ideal o extraeconómico ${ }^{18}$. En la doctrina italiana BRUNETTI define a la asociación en términos prácticamente idénticos ${ }^{19}$. Este concepto de asociación ha sido seguido por autores uruguayos $^{20}$ y argentinos ${ }^{21}$.

La organización se devela, entonces, en su verdadera importancia; la voluntad de ese ser, distinto a la simple suma de las voluntades individuales de sus miembros, se realiza y expresa a través de sus órganos ${ }^{22}$.

\section{La competencia orgánica y los procedimientos}

La idea de competencia y de procedimientos reglados para el funcionamiento de los órganos es consustancial al concepto de persona jurídica.

HAURIOU explica que, entre los miembros del grupo social interesado en la realización de la idea, se producen manifestaciones de comunión dirigidas por los órganos y regladas por ciertos procedimientos ${ }^{23}$. Las instituciones, afirma HAURIOU, viven gracias a operaciones jurídicas de gobierno y de administración repetidas y ligadas por procedimientos ${ }^{24}$.

Entre nosotros, CAJARVILle PELUFFo explica que la razón de ser del concepto de órgano es justificar que la actividad intelectual de una persona física se atribuya a un sujeto de derecho distinto ${ }^{25}$.

El concepto de competencia es, asimismo, consustancial al concepto de órgano. La competencia es el conjunto de facultades, funciones y atribuciones o poderes que la ley o el contrato le atribuye a un órgano.

SAYAGUÉS LASO se afilia a la opinión más generalizada según la cual el órgano comprende elcúmulo de funciones individualizadas y las personas llamadas a ejercerlas ${ }^{26}$. En el mismo orden de ideas, DelPiAzzo explica que ha de distinguirse entre el órgano y el cargo. Así, mientras el órgano tiene funciones definidas, el cargo dispone de funciones relativamente indeterminadas ${ }^{27}$.

18 Broseta Pont, Manuel, Manual de Derecho Mercantil, v. 1, 18 ed. a carago de Martínez Sanz, F. (Madrid: Tecnos, 2011), p. 275. Entre nosotros adoptan esta definición

19 Brunetti, Antonio, Tratado del derecho de las sociedades, trad. de Solá Cañízares, t.1, (Buenos Aires: Uthea, 1960), p. 5.

20 Rodríguez Olivera, Nuri Ethel y López Rodríguez, Carlos Eduardo, Manual de Derecho Comercial Uruguay, v. 4, t.1 (Montevideo: FCU, 2006), p. 175.

21 Alcorta, José, "La sociedad en la historia. Noción de sociedad comercial», en: Martorell, E.E. (Dir.), Tratado de Derecho Comercial, t 6, pp. 1-138 (Buenos Aires: La Ley, 2010), p. 26.

22 Barcia López, Arturo, Las personas jurídicas. Su responsabilidad civil por actos ilícitos (Buenos Aires: Valerio Abeledo, 1922), pp. 157, 215 y 216; Delpiazzo, Carlos Eduardo, Derecho Administrativo General, v.1 (Montevideo: Amalio M. Fernández, 2011), p. 227.

23 Hauriou, Maurice, Principios de derecho público y constitucional, traducción, estudio preliminar, notas y adiciones de Carlos Ruiz del Castillo [Madrid: Reus, 1927], p. 83).

24 Hauriou, Maurice, La teoría de la institución y de la fundación (Buenos Aires: Abeledo-Perrot, 1968), p. 40.

25 Cajarville Peluffo, op. cit., p. 572.

26 Sayagués Laso, op. cit., p. 181.

27 Delpiazzo, op. cit., p. 229. 
Cada órgano tiene, entonces, un ámbito de competencia que es exclusivo y excluyente. En la base de toda organización existe la separación de competencias, en un equivalente a la separación de poderes que se verifica en la organización estatal. Esta separación de competencias es lo que garantiza que un órgano no domine a otro ${ }^{28}$. MÉndEz explica que los órganos son titulares de una porción funcional determinada que se le atribuye por razón de especialización y división del trabajo. La competencia es, continúa MÉnDEZ, una creación técnica que corresponde al ejercicio de funciones que se determinan y asignan en cada acto de constitución orgánica, de ahí que cada órgano tenga funciones concretas y una competencia que, por ser proyección de éstas, se rige por igual solución ${ }^{29}$.

El concepto de competencia desempeña un papel equivalente al de capacidad de las personas físicas, de ahí que pueda afirmarse que las personas físicas gozan de capacidad y las personas jurídicas y sus órganos gozan de competencia. No obstante existir una importante diferencia de alcance entre un atributo y otro (la capacidad reconocida al ser humano lo habilita a desenvolverse en todo sentido, sin otros límites que los establecidos en la Constitución y las leyes [artículos 7 y 10 de la Constitución], mientras que los órganos actúan solamente en la zona que les fija la norma jurídica ${ }^{30}$ ), la consecuencia de la falta de este atributo provoca la misma consecuencia: la nulidad absoluta.

Sólo cuando el órgano actúa dentro de su competencia obliga a la persona jurídica. De otro modo, esos actos no tienen ninguna validez ${ }^{31}$ y las partes pueden comportarse tal como si el acto no se hubiera estipulado ${ }^{32}$. Así, los actos que los órganos realicen en exceso de sus competencias estarán viciados de nulidad y cuando incidan en la competencia de otro órgano supondrán, asimismo, una usurpación de competencias, lo cual es motivo de especial sanción en el Derecho Administrativo.

La nulidad de la resolución adoptada por un órgano francamente incompetente es plena, esto es, no produce ningún efecto. Sin embargo, esto no significa que no deba solicitarse un pronunciamiento judicial pues, como recuerda GAMARRA, nadie puede hacerse justicia por la propia mano ${ }^{33}$. Se tratará, éste, de un procedimiento ordinario y la sentencia que finalmente recaiga se limitará a constatar una nulidad preexistente ${ }^{34}$. Se encuentran legitimados para peticionar al juez la declaración de nulidad absoluta, no sólo quienes participaron en el acto en cuestión, sino todos los que resulten ajenos en tanto posean un interés legítimo ${ }^{35}$.

28 Hauriou, La teoría..., pp. 47 y 48.

29 Méndez, Aparicio, La teoría del órgano (Montevideo: Rosgal, 1949), pp. 28 y 29.

30 SAYAguÉs Laso, op. cit., p. 191.

31 Nuestra jurisprudencia declaró nulo un compromiso de compraventa otorgado por los integrantes de la Comisión Directiva de una Asociación en infracción al procedimiento establecido estatutariamente para la adquisición de ciertos bienes que ordenaba que tal decisión fuera considerada y resuelta por la Asamblea General (Sentencia del Tribunal de Apelaciones en lo Civil de $1^{\circ}$ turno de 25 de abril de 2014 (UY/jur7331772014). En igual sentido, no consideró obligada a una Asociación al pago de una suma de dinero a la que se habían comprometido los integrantes de la Comisión Directiva que desobedecieron el Estatuto y no llevaron el punto a la consideración de la Asamblea General (Sentencia del Tribunal de Apelaciones en lo Civil de $2^{\circ}$ turno de Montevideo, de 31 de marzo de 2004 (UY/JUR/223/2004).

32 Gamarna, Jorge, Tratado de Derecho Civil Uruguayo, t. XVI (Montevideo: s/e, 1974, p. 155.

33 GamarRa, op. cit., p. 161.

${ }^{34}$ Gamarra, id., ibíd.

${ }^{35}$ Gamarra, op. cit., pp. 167 y 168. 
Mientras tanto, los titulares delórgano tienen la obligación de defender su competen$\mathrm{cia}^{36}$ porque atribuir competencia es autorizar y, al mismo tiempo, imponer determinada $\operatorname{actividad}^{37}$.

\section{INTEGRACIÓN DEL DERECHO EN MATERIA DE ASOCIACIONES}

El artículo 21 del Código Civil carece de contenido suficiente para dilucidar muchos de los problemas jurídicos que eventualmente pueda afrontar una asociación civil. Es necesario, por tanto, integrar el faltante con otros cuerpos normativos.

Ciertas figuras son ciertamente afines a la asociación civil. Nos interesa tratar el caso de la sociedad comercial, contrato que tiene un cuerpo normativo extenso, dispuesto en la Ley 16.060/1989, de 4 de septiembre, de Sociedades Comerciales (LSC), que eventualmente podría completar el Derecho esbozado en el artículo 21 del Código Civil.

La afinidad entre la asociación y la sociedad comercial queda de manifiesto en las palabras de GARRIGUES DíAZ-CABAÑETE: «El tema de nuestro tiempo en el Derecho mercantil es la asociación. Asociación cuando el esfuerzo individual y aislado es insuficiente para la lucha con la competencia. Asociación también de las empresas sociales, que confluyen en más amplios organismos, donde se esfuma el primitivo sustrato personal a través de una sucesiva superposición de organizaciones colectivas» ${ }^{38}$. El autor se refiere a un "movimiento de asociación" y a la sociedad comercial como el «instrumento jurídico de conjunción de medios económicos que exceden la capacidad del hombre aislado» ${ }^{39}$.

Esta afinidad puede comprenderse de diferente manera. Tradicionalmente se ha considerado a la sociedad comercial como una especie dentro del concepto más amplio de la asociación ${ }^{40}$ o como una faceta de ésta ${ }^{41}$. Asociación civil y sociedad comercial se distinguirían por una nota específica: la de tener un fin económico destacado en el caso de la sociedad comercial, fin que estaría ausente en la asociación civil ${ }^{42}$, lo que ha dado en concretarse en el denominado «fin de lucro» ${ }^{43}$, esto es, la finalidad de repartir entre los socios las ganancias que se obtengan de la actividad ${ }^{44}$. Se agrega, además, que las asociaciones son ajenas a cualquier finalidad utilitaria. Poseen, en cambio, un propósito social desinteresado que impide la aplicación de la legislación comercial en ningún caso ${ }^{45}$.

36 SAYAgués Laso, op. cit., pp. 203 y 189.

37 Cajarville Peluffo, op. cit., p. 574, nota 14.

38 Garrigues Díaz-Cabañete, op. cit., p. 306.

39 Garrigues Díaz-Cabañete, íd.ibíd.

40 Garrigues Díaz-Cabañete, íd. ibíd.

41 Broseta Pont, op. cit., p. 275.

42 Garrigues Díaz-Cabañete, op. cit., p. 307.

43 BRunetTi, op. cit., p. 53.

${ }_{44}$ En la doctrina española a Menéndez-Menéndez, Aurelio, Estudios jurídicos y universitarios, t. 1 (Cizur Menor-Navarra: Thomson Reuters, 2015), p. 604. En la doctrina francesa véase a PILLET, Antoine, Des personnes morales (Paris: Recueil Sirey, 1914), p. 334. En la doctrina italiana véase a AscARell, Tulio, Sociedades y Asociaciones Comerciales (Buenos Aires: Ediar, 1947), p. 28. En la doctrina argentina véase a CAstillo Ramón, S., Curso de Derecho Comercial, t.3, 3ª ed. (Buenos Aires: Biblioteca Jurídica Argentina, 1935), p. 6.

45 Stanowsky, Marcos, Estudios de Derecho Comercial, t. 1 (Buenos Aires: Tipográfica Editora Argentina, 1950), pp. 226 у 227. 
Fuera de esta concepción tradicional, existe otra tendencia doctrinal que considera a la sociedad comercial comprendida en un concepto amplísimo de asociación, una suerte de "supraconcepto de sociedad», definido como agrupación voluntaria de personas para cualquier fin común caracterizada, no por el fin o causa, sino por la organización ${ }^{46}$.

En España se verifica este novedoso planteo del concepto de sociedad a partir de la revalorización del derecho constitucional de asociación y de la existencia de leyes que reconocen figuras asociativas a las que se le aplica, por mandato legal, la legislación societaria en forma supletoria. Por todo ello, a utores como Girón Tena, PAZ-Ares y SánchezCALERo concluyen que el ánimo de lucro no es esencial al concepto de sociedad ${ }^{47}$.

En Argentina, hace ya años, MALAgarRiga ponía el acento en la organización y desviaba la mirada de la finalidad de lucro y así afirmaba "Por ello, en resumen, consideramos en primer lugar, que si varias personas se agrupan para una finalidad común bajo la forma de sociedad anónima o de la sociedad de responsabilidad limitada, se tratará de sociedad y no de asociación y en segundo término, que tampoco cabrá negar el carácter de sociedad a una agrupación con finalidad común que no tome una de esas formas aunque se dé a las utilidades obtenidas otro destino que el de acrecentar el patrimonio de sus integrantes, siempre, bien entendido, que se hallen reunidos los elementos que distinguen la sociedad de otras situaciones jurídicas que se le asemejan, materia ésta de ardua dilucidación» ${ }^{48}$.

Entre nosotros predomina la posición tradicional y así se entiende que las asociaciones civiles son personas privadas sin fines de lucro, esto es, organizaciones que, basadas en el esfuerzo común de la agrupación humana que las constituye, tienen un fin desinteresado que excluye el económico. Justamente, éste es el elemento diferenciador entre las sociedades comerciales, organizaciones que, sean civiles o comerciales, persiguen siempre fines esencialmente económicos olucrativos ${ }^{49}$.

Como consecuencia de esta postura, dado que la sociedad y la asociación son organizaciones sustancialmente diferentes, no corresponde la integración de las omisiones estatutarias de una asociación con las normas de la LSC porque se trata de regímenes jurídicos incompatibles, lo que se percibe, en concreto, en la ausencia de distribución de utilidades al asociado a la finalización del ejercicio económico y la falta de un derecho a la participación en caso de retiro o exclusión del asociado ${ }^{50}$.

Asociación civil y sociedad comercial tendrían, no obstante la diferencia anotada, una importante similitud: la organización, organización que, en el caso de las sociedades anónimas, por ejemplo, desplaza al elemento contractual y las relaciones personales que

46 En la doctrina española véase Girón Tena, Sánchez Calero, Paz-Ares y Eizaguirre apud Vicent Chulí́, Vicent Chulí́, Francisco, Introducción al Derecho Mercantil, 23 edición, v. 1 (Valencia: tirant lo blanch, 2012), p. 441, Broseta Pont, op. cit., p. 276 y Langle, Emilio, Manual de Derecho Mercantil Español, t.1 (Barcelona: Bosch, 1950), p. 349.

47 Broseta Pont, op. cit., p. 276.

48 Malagarriga, Carlos, Tratado Elemental de Derecho Comercial, t. I (Buenos Aires: Tipográfica Editora Argentina, 1950), p. 169.

49 Gambino Crevani, op. cit., pp. 123; Rodríguez Olivera y López Rodríguez, Manual v.4, t., p. 175; Mezzera Álvarez, Rodolfo, Curso de Derecho Comercial, $2^{\text {a }}$ ed. (Montevideo: Medina, 1952), p. 21.

50 Rodríguez Olivera y López Rodríguez, íd. ibíd. En la Jurisprudencia, véase la Sentencia del Tribunal de Apelaciones en lo Civil de $2^{\circ}$ turno de Montevideo, de 31 de marzo de 2004 (UY/JUR/223/2004). 
nacen de él y, en su lugar, aparece la persona jurídica, entidad ideal que se presenta en el mundo jurídico como persona y que tiene una vida independiente de la de los socios ${ }^{51}$.

\section{CONCLUSIONES}

En nuestro país no existe una ley que establezca una regulación general para las asociaciones. En 1958 GAMBINO CREVANI explicaba que ello se debía a que tradicionalmente las cuestiones de estas personas jurídicas privadas sin finalidad de lucro rara vez trascendían lo económico.

Ciertamente hoy la situación ha cambiado. En la actualidad las asociaciones persiguen fines culturales, sociales o deportivos que no les impide obtener ganancias y aumentar su patrimonio, a veces de forma muy considerable. Trascienden, entonces, lo económico y algunas ganan mucho dinero. Lo que ocurre es que, a diferencia de las sociedades comerciales, el asociado no recibe ganancias. Toda la ganancia de la asociación se destina a la propia asociación que, indirectamente, favorecerá a sus asociados.

Asílas cosas, como primera conclusión, se echa en falta una ley general que establezca un mínimo de regulación, como acontece en otros países $^{52}$, pues la normativa sobre asociaciones civiles en nuestro país es parcial y se encuentra diseminada por todo nuestro ordenamiento jurídico, en normas de muy diverso rango.

En segundo lugar, las asociaciones civiles reconocidas por el Estado son personas jurídicas y, como tales, entes ideales que manifiestan su voluntad a través de sus órganos. Los órganos tienen un marco claro de funciones que cumplir y no pueden realizar otras tareas, ni invadir la esfera de competencia de otros órganos. Ello es garantía fundamental de la separación de poderes. Existen serias consecuencias para el obrar en contra del principio de competencia delórgano: de un lado, lo hecho por un órgano incompetente deriva en una nulidad absoluta por aplicación del artículo 1560 del Código Civil; de otro lado, el órgano desplazado debiera mantener su competencia puesto que proceder de otro modo compromete su responsabilidad en tanto la competencia de los órganos de una persona jurídica no ha de ser entendida como un derecho, sino como un deber.

En tercer lugar, el objeto social, deportivo o cultural de una asociación debiera ser entendido por oposición a objeto comercial, pero no como un objeto desinteresado. La asociación civil legítimamente puede pretender ganar dinero con su actividad y en ello no ha de verse reproche moral ni jurídico alguno. Lo que no ha de poder hacer la asociación civil es distribuir entre sus asociados los beneficios de esa actividad. Es por esta razón que la asociación civil es sustancialmente diferente de la sociedad comercial. No ha de olvidarse que la distribución de utilidades integra la definición de sociedad comercial, lo que es lo mismo que decir que no hay sociedad comercial sin pacto de distribución de utilidades. Dado

51 Garrigues Díaz-Cabañete, op. cit., pp. 307 y 308. En el mismo sentido, Alcorta, op. cit., p. 27.

52 Un buen ejemplo es España, país en que las asociaciones se encuentran reguladas en la Ley Orgánica 1/2002, de 22 de marzo. Esta Ley regula el derecho de asociación protegido en el artículo 22 de la Constitución española y refiere exclusivamente a las asociaciones en sentido estricto, esto es, las organizaciones que no tienen ánimo de lucro. Luego existen unas asociaciones que llevan a cabo actividades económicas. Éstas se encuentran reguladas por la Ley 5/2011, de 29 de marzo, de Economía social y que igual que las sociedades laborales, las empresas de inserción, los centros especiales de empleo y las cofradías de pescadores responden a ciertos principios de la denominada «economía social» (Vicent Chuliá, op. cit., pp. 441 y 442). 
esta diferencia de sustancia, no puede integrarse el derecho esbozado en el artículo 21 del Código Civil con la Ley 16.060 de Sociedades Comerciales. Llegado el caso, los vacíos habrán de colmarse atendiendo en primer lugar a los estatutos de la asociación y, en segundo, lugar, siguiendo el procedimiento impuesto en el artículo 16 del Código Civil.

\section{BIBLIOGRAFÍA CONSULTADA}

AlcorTA, José. «La sociedad en la historia. Noción de sociedad comercial», en: Martorell, E.E. (Dir.), Tratado de Derecho Comercial, t 6, pp. 1-138. Buenos Aires: La Ley, 2010.

Ascarelli, Tulio. Sociedades y Asociaciones Comerciales. Buenos Aires: Ediar, 1947.

BARCiA LóPez, Arturo. Las personas jurídicas. Su responsabilidad civil por actos ilícitos. Buenos Aires: Valerio Abeledo, 1922.

Berdaguer, Jaime. La sociedad civil jes sujeto de derecho? Montevideo: FCU, 2007.

Broseta Pont, Manuel. Manual de Derecho Mercantil, v. 1, 18 ed. a carago de Martínez Sanz, F. Madrid: Tecnos, 2011.

BRunetTi, Antonio. Tratado del derecho de las sociedades, trad. de Solá Cañízares, t.1. Buenos Aires: Uthea, 1960.

Cajarville Peluffo, Juan Pablo. Sobre Derecho Administrativo, t.1, $3^{\text {a }}$ ed. Montevideo: FCU, 2012. Castillo, Ramón S. Curso de Derecho Comercial, t.3, $3^{\text {a }}$ ed. Buenos Aires: Biblioteca Jurídica Argentina, 1935.

Couture, Eduardo Juan. Voz «personalidad jurídica» y voz «asociación», Vocabulario Jurídico. Montevideo: Biblioteca de Publicaciones Oficiales de la Facultad de Derecho y Ciencias Sociales de la Universidad de la República, 1960.

Delpiazzo, Carlos Eduardo. Derecho Administrativo General, v.1. Montevideo: Amalio M. Fernández, 2011.

Gamarna, Jorge. Tratado de Derecho Civil Uruguayo, t. XVI. Montevideo: s/e, 1974.

Gambino Crevani, Hugo. Personas jurídicas. Montevideo: Facultad de Derecho, 1958.

Garrigues Díaz-Cabañete, Joaquín. Curso de Derecho Mercantil, t. I, $7^{\text {a }}$ ed. Madrid: Aguirre, 1976. Hauriou, Maurice. La teoría de la institución y de la fundación. Buenos Aires: Abeledo-Perrot, 1968. Principios de derecho público y constitucional, traducción, estudio preliminar, notas y adiciones de Carlos Ruiz del Castillo. Madrid: Reus, 1927.

Langle, Emilio. Manual de Derecho Mercantil Español, t.1. Barcelona: Bosch, 1950.

Malagarriga, Carlos. Tratado Elemental de Derecho Comercial, t. I. Buenos Aires: Tipográfica Editora Argentina, 1950.

Martins, Daniel Hugo. Constitución de la República Oriental del Uruguay. Comentada, anotaday concordada, t. 1. Montevideo: La Ley, 2014.

Méndez, Aparicio. La teoría del órgano. Montevideo: Rosgal, 1949.

Menéndez-Menéndez, Aurelio. Estudios jurídicos y universitarios, t. 1. Cizur Menor-Navarra: Thomson Reuters, 2015.

Mezzera Álvarez, Rodolfo. Curso de Derecho Comercial, $2^{\text {a }}$ ed. Montevideo: Medina, 1952.

Pillet, Antoine. Des personnes morales. Paris: Recueil Sirey, 1914.

Rodríguez Olivera, Nuri Ethel, y LóPez Rodríguez, Carlos Eduardo. Manual de Derecho Comercial Uruguay, v. 4, t.1. Montevideo: Fundación de Cultura Universitaria, 2006.

Sayagués Laso, Enrique. Tratado de Derecho Administrativo, t.1 Montevideo: s/e, 1963.

Stanowsky, Marcos. Estudios de Derecho Comercial, t. 1. Buenos Aires: Tipográfica Editora Argentina, 1950.

Vicent Chulí, Francisco. Introducción al Derecho Mercantil, 23 edición, v. 1. Valencia: tirant lo blanch, 2012.

Fecha de recepción: 23 noviembre 2017.

Fecha de aceptación: 1 de marzo 2018. 
